\title{
Interactions impliquant des médicaments à base d'Hypericum: mise à jour des informa- tions sur les médicaments
}

\author{
J. Djonova, R. Stoller
}

Correspondance:

Dr Julia Djonova

Swissmedic, Institut suisse

des produits thérapeutiques Division médicaments complémentaires et phytothérapeutiques Hallerstrasse 7

CH-3000 Berne 9

E-mail:

julia.djonova@swissmedic.ch
Ces dernières années, des preuves de plus en plus évidentes des interactions de Hypericum perforatum (millepertuis) avec un grand nombre de médicaments ont été rapportées. Des interactions cliniquement pertinentes ont en effet été observées lors de la prise d'Hypericum avec des anticoagulants oraux, des immunosuppresseurs (ciclosporine, tacrolimus), des contraceptifs oraux, de la digoxine et certains autres médicaments antiviraux. De plus, des interactions avec d'autres médicaments tels que certains cytostatiques et la méthadone ont récemment été mises en évidence. Enfin, certains cas annoncés dans le cadre de la surveillance du marché ont contribué à compléter les données disponibles sur des interactions connues, par exemple avec des contraceptifs hormonaux ou des antiviraux. Enfin, pour faire le point sur les toutes dernières découvertes faites en la matière, Swissmedic a lancé une évaluation des données disponibles et une mise à jour des informations destinées aux patients et des informations professionnelles des préparations à base d'Hypericum.

De nombreuses préparations à base de millepertuis sont autorisées en Suisse dans le traitement de la baisse de l'humeur, de la labilité de l'humeur et des états de tension. Et il n'est pas rare qu'elles soient prises en même temps que d'autres médicaments à l'insu du médecin. Une étude réalisée en Allemagne a ainsi montré que 1 patient sur 14 admis à l'hôpital prend à son arrivée une préparation à base d'Hypericum, mais sans que les médecins le sachent; de plus, 1 patient sur 21 reçoit des médicaments qui peuvent interagir avec le millepertuis [1]. Les interactions sont vraisemblablement dues à une activation du cytochrome P450 (surtout du CYP3A4) et/ou à une activation des protéines de transport (glycoprotéine P), ce qui entraîne une baisse de la concentration plasmatique du principe actif influencé par l'Hypericum. L'effet est particulièrement marqué avec des substances ayant un métabolisme présystémique important. Ainsi, l'effet thérapeutique de nombreux médicaments est réduit voire supprimé. Soulignons également que des interactions pharmacodynamiques sont possibles, par exemple avec des médicaments sérotoninergiques, dont certains antidépresseurs. Elles seraient dues à des effets sérotoninergiques renforcés, qui peuvent causer l'apparition des symptômes du syndrome sérotoninergique plutôt que du syndrome dans son ensemble. Ce syndrome se manifeste au travers de troubles moteurs (myoclonies, tremblements, hyperréflexie), de troubles psychiatriques (confusion, agitation) et de symptômes autonomes (hypersudation, diarrhée, tachycardie).

Eu égard aux graves conséquences cliniques potentielles, il convient d'ajouter dans les textes d'information sur les préparations contenant de l'Hypericum les interactions avec les médicaments/groupes de médicaments suivants:

- Cytostatiques (Imatinib, Irinotecan). Dans le cadre d'une étude d'interactions de deux semaines, on a observé chez les sujets de recherche une diminution significative de la concentration plasmatique et de l'AUC de l'Imatinib de respectivement 43 et $30 \%$ ainsi qu'une augmentation de la clairance de cette substance [2,3]. Ces résultats ont été confirmés par des observations concordantes faites dans une étude ouverte incluant 5 patients cancéreux à qui de l'Irinotecan et de l'Hypericum ont été administrés simultanément [4]. Compte tenu des interactions possibles, les préparations à base de millepertuis ne devraient pas être prises avant et pendant l'administration de cytostatiques dont le métabolisme fait intervenir les enzymes CYP et la P-glycoprotéine.

- Méthadone. On a observé chez quatre patients traités par la méthadone à qui on a administré simultanément une préparation à base de millepertuis, une forte baisse de la concentration plasmatique en méthadone (de 47\%) [5]. Des annonces spontanées d'effets secondaires ont également fait état de symptômes correspondants (perte d'efficacité). Enfin, l'arrêt de la prise d'Hypericum peut faire monter le taux thérapeutique de méthadone à un niveau toxique. 
Les mises en garde susmentionnées concernant d'autres groupes de médicaments avec lesquels les interactions sont connues ont été précisées. Ainsi, l'administration concomitante de préparations à base de millepertuis avec des anticoagulants coumariniques, avec certains immunosuppresseurs (ciclosporine, tacrolimus, sirolimus) et des antirétroviraux (inhibiteurs de la protéase du VIH-1 ou inhibiteurs nucléosidiques de la transcriptase inverse) est considérée comme contre-indiquée.

Le millepertuis ne doit en outre pas être administré, ou sous surveillance médicale étroite, avec de la théophylline et des analogues ou avec des antidépresseurs (risque de syndrome sérotoninergique).

Il convient également de rappeler que l'arrêt de la prise d'une préparation à base de millepertuis en cas d'association accidentelle avec l'un des médicaments précités doit se faire sous surveillance médicale. En effet, la dose de principe actif qui interagit avec le millepertuis doit souvent être adaptée en surveillant étroitement son effet (p.ex. taux normalisé international [INR] avec les anticoagulants) et ses concentrations plasmatiques (ciclosporine, digoxine). Certains auteurs recommandent par conséquent un arrêt par palier de l'Hypericum.

La littérature scientifique de même que les annonces spontanées d'effets indésirables font état de cas isolés de grossesse non désirée [6] ou de survenue de métrorragies en cas de prise concomitante de préparations à base de millepertuis et d'une contraception hormonale. Les annonces récentes concernent aussi bien les contraceptifs oraux que d'autres types de contraceptifs hormonaux (implants, transdermiques, intra-utérins et vaginaux) avec lesquels la prudence est donc de rigueur. Enfin, des interactions avec d'autres médicaments (p.ex. certains antiépileptiques, hypoglycémiants ou hormones stéroïdiennes) ne peuvent être exclues.

D'autres réactions ont en outre été rapportées, telles que des cas isolés de crise d'hypertension lors d'un traitement par millepertuis, qui ont pu être expliquées par l'inhibition de la monoamine oxydase (syndrome noradrénergique), bien que d'autres mécanismes puissent être impliqués. Il est donc important que les professionnels de la santé demandent à leurs patients s'ils prennent une préparation à base de millepertuis avant de leur prescrire des médicaments. S'il n'est pas possible d'arrêter la préparation à base de millepertuis, il convient d'envisager une adaptation de la posologie du médicament prescrit ou d'assurer une surveillance clinique étroite.

Les annonces spontanées d'effets indésirables ont joué un rôle clé dans la mise au jour des interactions du millepertuis. Mais pour pouvoir étayer davantage encore les nouvelles dispositions prises et déceler d'autres problèmes éventuels, nous prions les professionnels de santé d'annoncer les interactions ou les autres effets indésirables suspectés en remplissant dûment le formulaire jaune prévu à cet effet, qui doit être envoyé à l'un des centres régionaux de pharmacovigilance. Un exemplaire de ce formulaire est joint au présent document. Mais il peut également être téléchargé sur le site Internet de Swissmedic (www.swissmedic.ch $\rightarrow$ Surveillance du marché $\rightarrow$ Pharmacovigilance $\rightarrow$ Répertoire général Pharmacovigilance $\rightarrow$ Formulaire d'annonce) ou être commandé auprès de l'Institut.

\section{Références}

1 Martin-Facklam M, Rieger K, Riedel KD, Burhenne J, Walter-Sack I, Haefeli WE. Undeclared exposure to St. John's Wort in hospitalized patients. Br J Clin Pharmacol 2004;58:437-41.

2 Frye RF, Fitzgerald SM, Lagattuta TF, Hruska MW, Egorin MJ. Effect of St John's wort on imatinib mesylate pharmacokinetics. Clin Pharmacol Ther 2004;76:323-9.

3 Smith P, Bullock JM, Booker BM, Haas CE, Berenson CS, Jusko WJ. The influence of St. John's wort on the pharmacokinetics and protein binding of imatinib mesylate. Pharmacotherapy 2004;24(11):1508-14.

4 Mathijssen RH, Verweij J, de Bruijn P, Loos WJ, Sparreboom A. Effects of St. John's wort on irinotecan metabolism. J Natl Cancer Inst 2002; 94(16):1247-9.

5 Eich-Höchli D, Oppliger R, Powell Goday K, Baumann P, Eap CB. Methadone maintenance treatment and St. John's Wort - a case report. Pharmacopsychiatry 2003;36:35-7.

6 Schwarz UI, Buschel B, Kirch W. Unwanted pregnancy on self-medication with St John's wort despite hormonal contraception. Br J Clin Pharmacol 2003;55(1):112-3. 